Portland State University

PDXScholar

\title{
Space-time CFOSLS Methods with AMGe Upscaling
}

Martin Neumüller

Johannes Kepler University of Linz

Panayot Vassilevski

Portland State University

Umberto E. Villa

University of Texas at Austin

Follow this and additional works at: https://pdxscholar.library.pdx.edu/mth_fac

Part of the Mathematics Commons

Let us know how access to this document benefits you.

\section{Citation Details}

Neumüller M., Vassilevski P.S., Villa U.E. (2017) Space-Time CFOSLS Methods with AMGe Upscaling. In: Lee CO. et al. (eds) Domain Decomposition Methods in Science and Engineering XXIII. Lecture Notes in Computational Science and Engineering, vol 116. Springer, Cham

This Pre-Print is brought to you for free and open access. It has been accepted for inclusion in Mathematics and Statistics Faculty Publications and Presentations by an authorized administrator of PDXScholar. Please contact us if we can make this document more accessible: pdxscholar@pdx.edu. 


\title{
Space-time CFOSLS Methods with AMGe Upscaling
}

\author{
Martin Neumüller ${ }^{1}$, Panayot S. Vassilevski ${ }^{2}$, and Umberto E. Villa ${ }^{3}$
}

\begin{abstract}
This work considers the combined space-time discretization of time-dependent partial differential equations by using first order least square methods. We also impose an explicit constraint representing space-time mass conservation. To alleviate the restrictive memory demand of the method, we use dimension reduction via accurate element agglomeration AMG coarsening, referred to as AMGe upscaling. Numerical experiments demonstrating the accuracy of the studied AMGe upscaling method are provided.
\end{abstract}

\section{Introduction}

In this paper we explore a robust approach to derive combined space-time discretization methods for two classes (parabolic and hyperbolic) of timedependent PDEs. We use the popular FOSLS (first order systems leastsquares) approach (cf., e.g., Cai et al. [1994] or Carey et al. [1995]) ) treating time as an additional space variable and, in addition, we prescribe a space-time divergence equation as a constraint in order to maintain certain space-time mass conservation (following, e.g., Adler and Vassilevski [2014]).

More specifically, our approach is applied to the following model problem

\footnotetext{
[1] Johannes Kepler University Linz, Institute of Computational Mathematics, Altenberger Straße 69, 4040 Linz, Austria neumueller@numa.uni-linz.ac.at. . [2] Center for Applied Scientific Computing, Lawrence Livermore National Laboratory, P.O. Box 808, L-561, Livermore, CA 94551, U.S.A. panayot@llnl.gov . [3] The University of Texas at Austin, Institute for Computational Engineering and Sciences (ICES), 201 E. 24th Street, Stop C0200, Austin, Texas 78712-0027, U.S.A. uvilla@ices.utexas.edu

0 This work was performed under the auspices of the U.S. Department of Energy by Lawrence Livermore National Laboratory under Contract DE-AC52-07NA27344. The work was partially supported by ARO under US Army Federal Grant \# W911NF-15-1-0590.
} 


$$
\frac{\partial S}{\partial t}+\operatorname{div}(\mathcal{L}(S))=q_{0}(\mathbf{x}, t), \quad \mathbf{x} \in \Omega \subset \mathbb{R}^{d}, t \in(0, T),
$$

where $\mathcal{L}$ is at most a first-order differential operator with respect to the space variable $\mathbf{x}$ only. At $t=0$ we impose an initial condition $S=S_{0}$ and on $\partial \Omega$ for all $t \in(0, T)$ we apply some appropriate boundary conditions (if any). More specifically we consider differential operators of the form

$$
\mathcal{L}(S):=-k \nabla_{\mathbf{x}} S \quad \text { and } \quad \mathcal{L}(S):=f(S) \mathbf{u}(\cdot)
$$

for respectively parabolic and hyperbolic problems, as explained in more details in Section 4 and 5.

\section{Space-time Constrained First Order System Least Squares}

Problem (1) can be rewritten as a first order system by introducing the "flux" variable $\boldsymbol{\sigma}:=[\mathcal{L}(S) ; S]^{\top}$ as

$$
\begin{aligned}
\boldsymbol{\sigma}-\left[\begin{array}{c}
\mathcal{L}(S) \\
S
\end{array}\right] & =0 \\
\operatorname{div}_{\mathbf{x}, t} \boldsymbol{\sigma} & =q_{0}
\end{aligned}
$$

where $\operatorname{div}_{\mathbf{x}, t}$ is the $d+1$-dimensional space-time divergence operator. We then introduce the FOSLS functional as

$$
J(\boldsymbol{\sigma}, S)=\left\|\boldsymbol{\sigma}-\left[\begin{array}{c}
\mathcal{L}(S) \\
S
\end{array}\right]\right\|_{0, K^{-1}}^{2}+\left\|q_{0}-\operatorname{div}_{\mathbf{x}, t} \boldsymbol{\sigma}\right\|_{0}^{2},
$$

where $K=K(\mathbf{x}) \in \mathbb{R}^{(d+1) \times(d+1)}$ is a symmetric and positive definite coefficient matrix and $\|\cdot\|_{0}\left(\|\cdot\|_{0, K^{-1}}\right)$ denotes the (weighted) $L_{2}\left(\Omega_{T}\right)$-norm with respect to the space-time domain $\Omega_{T}:=\Omega \times(0, T)$. A constrained leastsquare version of (2) is given by minimizing the functional $J(\boldsymbol{\sigma}, S)$ under the constraint which is given by the conservation equation

$$
\left(\operatorname{div}_{\mathbf{x}, t} \boldsymbol{\sigma}, w\right)=\left(q_{0}, w\right) \text { for all } w \in L_{2}\left(\Omega_{T}\right) .
$$

Here we denote with $(\cdot, \cdot)$ the inner product with respect to $L_{2}\left(\Omega_{T}\right)$. First order optimality conditions for the constrained minimization problem lead to the system of variational equations: Find $\boldsymbol{\sigma} \in H\left(\operatorname{div}_{\mathbf{x}, t} ; \Omega_{T}\right), S \in V$ and $\mu \in L_{2}\left(\Omega_{T}\right)$, such that 


$$
\begin{array}{ll}
(\boldsymbol{\sigma}, \boldsymbol{\psi})_{K^{-1}}+\left(\operatorname{div}_{\mathbf{x}, t} \boldsymbol{\sigma}, \operatorname{div}_{\mathbf{x}, t} \boldsymbol{\psi}\right)-\left(\left[\begin{array}{c}
\mathcal{L}(S) \\
S
\end{array}\right], \boldsymbol{\psi}\right)_{K^{-1}}+\left(\mu, \operatorname{div}_{\mathbf{x}, t} \boldsymbol{\psi}\right) & =\left(q_{0}, \operatorname{div}_{\mathbf{x}, t} \boldsymbol{\psi}\right), \\
-\left(\boldsymbol{\sigma},\left[\begin{array}{c}
\mathcal{L}(\phi) \\
\phi
\end{array}\right]\right)_{K^{-1}}+\left(\left[\begin{array}{c}
\mathcal{L}(S) \\
S
\end{array}\right],\left[\begin{array}{c}
\mathcal{L}(\phi) \\
\phi
\end{array}\right]\right)_{K^{-1}} & =0 \\
\left(\operatorname{div}_{\mathbf{x}, t} \boldsymbol{\sigma}, w\right) & =\left(q_{0}, w\right)
\end{array}
$$

holds for all $\boldsymbol{\psi} \in H\left(\operatorname{div}_{\mathbf{x}, t} ; \Omega_{T}\right)$, all $\phi \in V$ and all $w \in L_{2}\left(\Omega_{T}\right)$. Here $V$ denotes an appropriate function space for the unknown $S$, such that $\mathcal{L}: V \rightarrow L^{2}$ is a bounded operator. In a straightforward manner we obtain the finite element discretization of the CFOSLS system (3) by using appropriate finite dimensional spaces, i.e. we use $\boldsymbol{\sigma}_{h} \in \mathbf{R}_{h} \subset H\left(\operatorname{div}_{\mathbf{x}, t} ; \Omega_{T}\right), S_{h} \in V_{h} \subset V$ and $\mu_{H} \in W_{H} \subset L_{2}\left(\Omega_{T}\right)$. Note that the Lagrangian multiplier $\mu_{H}$ belongs to the space $W_{H}$ of discontinuous piecewise polynomials defined on a coarser mesh $\mathcal{T}_{H}$ (the lowest order being piecewise constants). The fine mesh $\mathcal{T}_{h}$ is constructed by performing one uniform refinement of $\mathcal{T}_{H}$. This choice leads to a relaxed Petrov-Galerkin discretization of the mass conservation equation and prevents overconstraining the resulting system. A relevant error analysis of the above discretization has been presented in Adler and Vassilevski [2014]. Finally, using appropriate basis functions for the discrete function spaces, we obtain the system of linear equations for the saddle point problem

$$
\left[\begin{array}{ccc}
A & B^{\top} & D^{\top} \\
B & C & 0 \\
D & 0 & 0
\end{array}\right]\left[\begin{array}{c}
\boldsymbol{\sigma}_{h} \\
\mathbf{S}_{h} \\
\boldsymbol{\mu}_{H}
\end{array}\right]=\left[\begin{array}{c}
f_{h} \\
0 \\
g_{H}
\end{array}\right] .
$$

\section{AMGe Upscaling}

The AMGe (element agglomeration) coarsening has been developed at LLNL, originally to derive hierarchies of finite element spaces for designing multigrid solvers for bilinear forms corresponding to an entire de Rham sequence of spaces $\left(H^{1}\right.$-conforming, $H$ (curl)-conforming, and $H$ (div)-conforming), (Pasciak and Vassilevski [2008]), and more recently (Lashuk and Vassilevski [2012, 2014]) to ensure that these hierarchies of spaces have guaranteed approximation properties. Such spaces are hence suitable to construct accurate coarse discretizations and can be used as a tool for dimension reduction, also refereed to as numerical upscaling.

The CFOSLS space-time discretization approach leads to saddle-point systems involving function spaces in the divergence constraint that are $H$ (div)conforming. This allows to solve combined space-time problems up to 2 space dimensions using the existing AMGe upscaling framework for 3D RaviartThomas elements. The goal in the near future is to extend this framework to 4D Raviart-Thomas analogs. This paper, as a first step, demonstrates the feasibility of the AMGe upscaling approach applied to combined space-time 
discretization that is both accurate, mass-conservative and achieving reasonable dimension reduction, which makes the expensive direct space-time approach (applied on the fine grid) feasible at coarser upscaled levels.

In the next sections we study the presented approach in detail for the two differential operators introduced in the beginning of this work. The finite element library MFEM (MFEM) is used to assemble the discretized systems which are then solved using the algebraic multigrid solvers (AMG) in hypre (HYPRE).

\section{Parabolic problem}

Here we choose the differential operator $\mathcal{L}(S):=-k \nabla_{\mathbf{x}} S$, where $k=k(\mathbf{x})$ is a given positive coefficient. For simplicity, we use homogeneous Dirichlet boundary conditions on $\partial \Omega$ for all $t \in(0, T)$. For the variational problem (3) we then introduce the weight

$$
K=\left[\begin{array}{cc}
k I_{d} & 0 \\
0 & 1
\end{array}\right] .
$$

A natural space for the unknown $S$ is then given by $V=L_{2}\left(0, T, H_{0}^{1}(\Omega)\right)$. For the discretization, we use a standard conforming subspace $V_{h} \subset V$ consisting of piecewise Lagrangian polynomials which are globally continuous. We then solve the discretized saddle-point problem (4) by using the MINRES method with the block diagonal preconditioner

$$
\hat{P}=\left[\begin{array}{ccc}
\hat{A} & 0 & 0 \\
0 & \hat{C} & 0 \\
0 & 0 & \hat{W}
\end{array}\right],
$$

where $\hat{A}$ denotes the auxiliary space AMG solver for $H$ (div)-problem applied to the matrix $A$ (HypreADS, Kolev and Vassilevski [2012]), $\hat{C}$ is a standard AMG preconditioner for $C$ (BoomerAMG, HYPRE), and $\hat{W}$ represents the diagonal of the $L_{2}\left(\Omega_{T}\right)$ mass matrix $W$.

Example 1. In this example we let $\Omega=(0,1)^{2}, T=1$ and $k \equiv 1$. The exact solution is given by $u\left(x_{1}, x_{2}, t\right)=e^{-t} \sin \left(\pi x_{1}\right) \sin \left(\pi x_{2}\right)$.

The initial - fine - space-time mesh (level 0 ) is an unstructured tetrahedral mesh with 490, 200 elements. We use graph partitioning algorithms (Karypis and Kumar [1998]) to construct the agglomerated space-time meshes shown in Figure 1. For the discretization, we use lowest order finite element spaces on the fine grid and then we construct the hierarchy of coarse spaces as explained in Section 3. Table 1 reports the errors with respect to the exact solution. We observe that the upscaling procedure allows to dramatically reduce the 
number of unknowns maintaining reasonable good approximations, see also Figure 1 .

\begin{tabular}{c|rr||cc|cc|r} 
level & elements & dof & $\left\|S-S_{H}\right\|_{0}$ & $\left\|\boldsymbol{\sigma}-\boldsymbol{\sigma}_{H}\right\|_{0}$ & $\left\|u_{h}-u_{H}\right\|_{0}\left\|\boldsymbol{\sigma}_{h}-\boldsymbol{\sigma}_{H}\right\|_{0}$ & iter \\
\hline 0 & 490,200 & $1,579,808$ & $3.4360 \mathrm{E}-03$ & $2.4217 \mathrm{E}-02$ & - & - & 107 \\
1 & 7,700 & 218,089 & $6.2509 \mathrm{E}-03$ & $3.2351 \mathrm{E}-02$ & $2.0985 \mathrm{E}-03$ & $3.5408 \mathrm{E}-02$ & 80 \\
2 & 1,043 & 59,085 & $2.5489 \mathrm{E}-02$ & $7.5482 \mathrm{E}-02$ & $8.3829 \mathrm{E}-03$ & $1.0854 \mathrm{E}-01$ & 102 \\
3 & 179 & 12,366 & $8.1318 \mathrm{E}-02$ & $1.7308 \mathrm{E}-01$ & $2.6544 \mathrm{E}-02$ & $2.5752 \mathrm{E}-01$ & 60 \\
4 & 39 & 3,127 & $2.3470 \mathrm{E}-01$ & $3.7018 \mathrm{E}-01$ & $7.6846 \mathrm{E}-02$ & $5.5365 \mathrm{E}-01$ & 34 \\
5 & 8 & 635 & $3.0685 \mathrm{E}-01$ & $5.1457 \mathrm{E}-01$ & $1.0064 \mathrm{E}-01$ & $7.7024 \mathrm{E}-01$ & 27 \\
\hline
\end{tabular}

Table 1 Numerical errors for different agglomeration levels for Example 1.

\section{Hyperbolic problem}

Here we consider the differential operator $\mathcal{L}(S):=f_{0}\left(S_{*}\right) S \mathbf{u}(\cdot)$, with the given velocity field $\mathbf{u}$ (satisfying $\mathbf{u} \cdot \mathbf{n}_{\mathbf{x}}=0$ on $\partial \Omega$ ) and the given positive function $f_{0}=f_{0}\left(S_{*}\right)$. Such equations can be used, for example, to model the evolution in time of water or gas saturation in an oil reservoir. We then introduce the weight

$$
K=K\left(S_{*}\right)=\left[\begin{array}{cc}
f_{0}\left(S_{*}\right) I_{d} & 0 \\
0 & 1
\end{array}\right] \quad \text { which gives } \boldsymbol{\sigma}=K\left(S_{*}\right)\left[\begin{array}{l}
\mathbf{u} \\
1
\end{array}\right] S .
$$

A natural setting for $S$ is given by $V=L_{2}\left(\Omega_{T}\right)$. Using the second equation of (3) we can eliminate the unknown $S$ and we obtain the reduced system for $\boldsymbol{\sigma}$ and the Lagrange multiplier $\mu$ : Find $\boldsymbol{\sigma} \in H\left(\operatorname{div} ; \Omega_{T}\right)$ and $\mu \in L_{2}\left(\Omega_{T}\right)$, such that

$$
\begin{aligned}
\left(\left(K^{-1}-\delta_{K}^{-1}\left[\begin{array}{c}
\mathbf{u} \\
1
\end{array}\right]\left[\begin{array}{l}
\mathbf{u} \\
1
\end{array}\right]^{\top}\right) \boldsymbol{\sigma}, \boldsymbol{\psi}\right)+(\mu, \operatorname{div} \boldsymbol{\psi}) & =0, \\
(\operatorname{div} \boldsymbol{\sigma}, w) & =(q, w)
\end{aligned}
$$

holds for all $\boldsymbol{\psi} \in H\left(\operatorname{div} ; \Omega_{T}\right)$ and for all $w \in L_{2}\left(\Omega_{T}\right)$. Here $\delta_{K} \in \mathbb{R}$ is given by

$$
\delta_{K}=\left[\begin{array}{c}
\mathbf{u} \\
1
\end{array}\right]^{\top} K\left[\begin{array}{l}
\mathbf{u} \\
1
\end{array}\right] \quad \text { and further } \quad S=\delta_{K}^{-1}\left[\begin{array}{c}
\mathbf{u} \\
1
\end{array}\right]^{\top} \boldsymbol{\sigma} .
$$

It can be shown that the matrix $K^{-1}-\delta_{K}^{-1}\left[\begin{array}{l}\mathbf{u} \\ 1\end{array}\right]\left[\begin{array}{l}\mathbf{u} \\ 1\end{array}\right]^{\top}$ in (5) is positive definite on the nullspace of the divergence operator, if $\operatorname{div}_{\mathbf{x}}\left(f_{0}\left(S_{*}\right) \mathbf{u}\right) \geq 0$ in $\Omega$ and $\mathbf{u} \cdot \mathbf{n}_{\mathbf{x}}=0$ on $\partial \Omega$. 


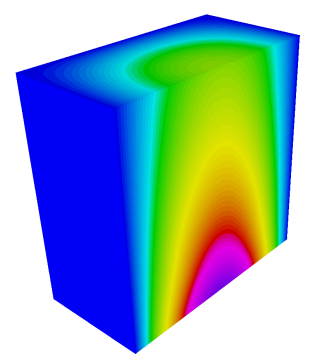

Numerical solution $S_{h}$

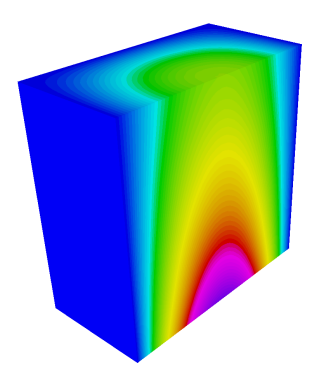

Numerical solution $S_{h}$

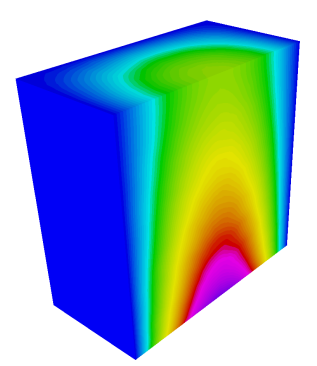

Numerical solution $S_{h}$

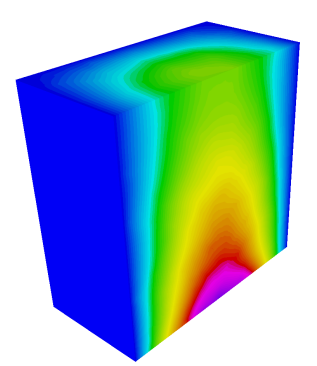

Numerical solution $S_{h}$

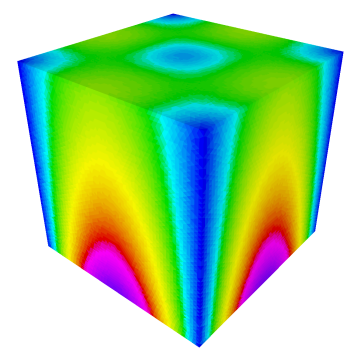

Numerical solution $\left|\boldsymbol{\sigma}_{h}\right|$

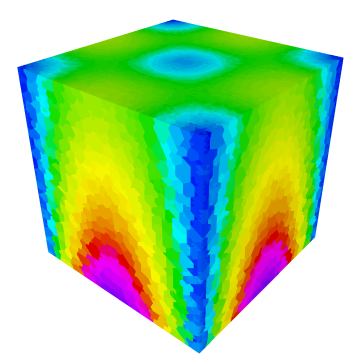

Numerical solution $\left|\boldsymbol{\sigma}_{h}\right|$

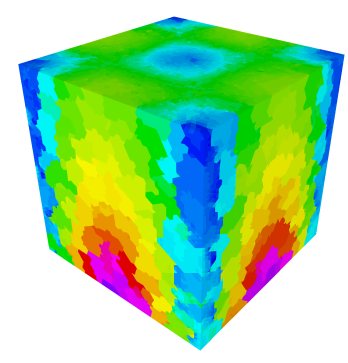

Numerical solution $\left|\boldsymbol{\sigma}_{h}\right|$

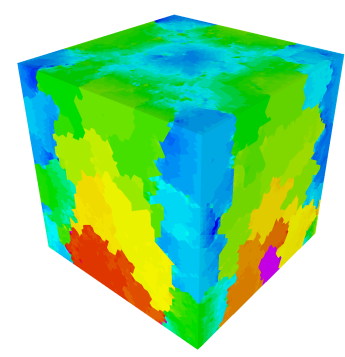

Numerical solution $\left|\boldsymbol{\sigma}_{h}\right|$

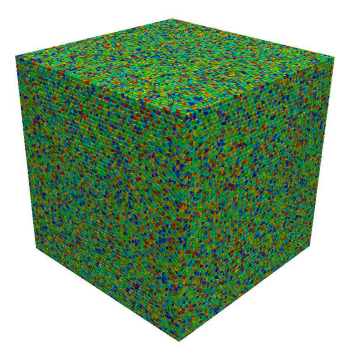

Agglomerated mesh on level 0

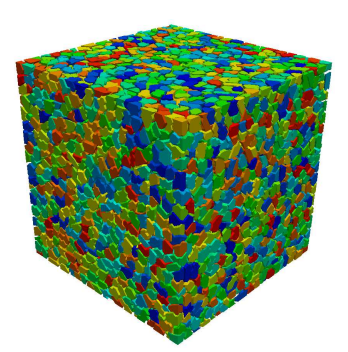

Agglomerated mesh on level 1

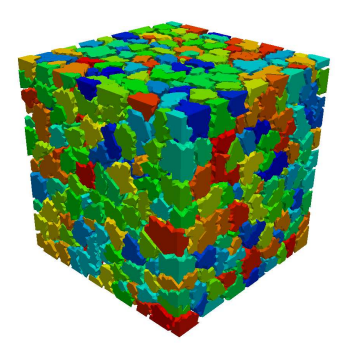

Agglomerated mesh on level 2

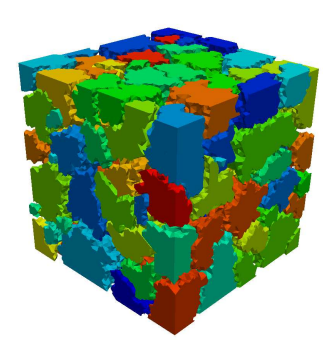

Agglomerated mesh on level 3

Fig. 1 Numerical solutions and agglomerated meshes for different levels (Example 1). 
Example 2. In this example we consider $\Omega=\left\{\mathbf{x} \in \mathbb{R}^{2}:|\mathbf{x}|<1\right\}, T=2$, $f_{0}\left(S_{*}\right) \equiv 1$ and $q_{0} \equiv 0$ with the velocity function and the initial condition

$$
\mathbf{u}\left(x_{1}, x_{2}, t\right)=\left[\begin{array}{c}
-x_{2} \\
x_{1}
\end{array}\right] \quad \text { and } \quad S_{0}\left(x_{1}, x_{2}\right)=e^{-100\left[\left(x_{1}-0.5\right)^{2}+x_{2}^{2}\right]} .
$$

For the discretization we use Raviart-Thomas pairs $\mathbf{R}_{h}, W_{h}$ for $\boldsymbol{\sigma}$ and the Lagrange multiplier $\mu$. The initial fine mesh (an unstructured tetrahedral mesh with 1,315, 708 elements) and the agglomerated meshes are shown in Figure 2. Table 2 shows (similarly to what already observed for the parabolic example) that upscaling allows to achieve both effective dimension reduction and good approximation of the fine grid solution (level 0). The divergence free solver Christensen et al. [2015] allows for the robust solution of the discretized saddle point problem at each level as shown by the number of iterations reported in Table 2.

\begin{tabular}{c|rr||cc|r} 
level & elements & dof & $\left\|\boldsymbol{\sigma}_{h}-\boldsymbol{\sigma}_{H}\right\|_{0}$ & $\left\|\mu_{h}-\mu_{H}\right\|_{0}$ & iter \\
\hline 0 & $1,315,708$ & $3,970,948$ & - & - & 39 \\
1 & 164,495 & $1,636,016$ & $1.1665 \mathrm{E}-03$ & $1.2176 \mathrm{E}-09$ & 39 \\
2 & 21,009 & 495,815 & $5.0647 \mathrm{E}-03$ & $2.2788 \mathrm{E}-04$ & 33 \\
3 & 3,215 & 99,004 & $9.1879 \mathrm{E}-03$ & $4.6800 \mathrm{E}-04$ & 24 \\
4 & 684 & 22,324 & $1.0483 \mathrm{E}-02$ & $5.6677 \mathrm{E}-04$ & 19 \\
5 & 200 & 8,041 & $1.2115 \mathrm{E}-02$ & $7.1052 \mathrm{E}-04$ & 16 \\
\hline
\end{tabular}

Table 2 Numerical errors for different agglomeration levels for Example 2.

\section{References}

J. H. Adler and P. S. Vassilevski. Error Analysis for Constrained First-Order System least-Squares Finite-Element Methods. SIAM J. Sci. Comput., 36 (3):A1071-A1088, 2014.

Z. Cai, R. Lazarov, T. A. Manteuffel, and S. F. McCormick. First-order system least squares for second-order partial differential equations. I. SIAM J. Numer. Anal., 31(6):1785-1799, 1994.

G. F. Carey, A. I. Pehlivanov, and P. S. Vassilevski. Least-squares mixed finite element methods for non-selfadjoint elliptic problems. II. Performance of block-ILU factorization methods. SIAM J. Sci. Comput., 16(5):1126-1136, 1995.

M. Christensen, U. Villa, and P. S. Vassilevski. Multilevel Techniques Lead to Accurate Numerical Upscaling and Scalable Robust Solvers for Reservoir Simulation. SPE Reservoir Simulation Symposium, 23-25 February, Houston, Texas, USA, SPE-173257-MS, 2015. 

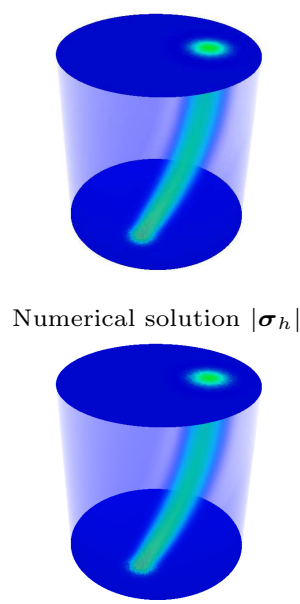

Numerical solution $\left|\boldsymbol{\sigma}_{h}\right|$

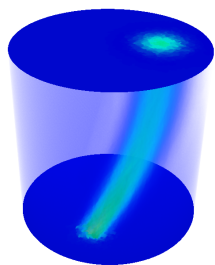

Numerical solution $\left|\boldsymbol{\sigma}_{h}\right|$

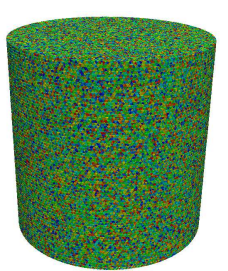

Agglomerated mesh on level 0

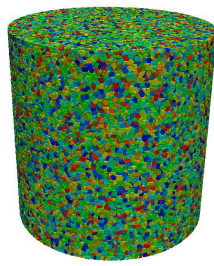

Agglomerated mesh on level 1

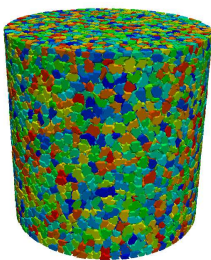

Agglomerated mesh on level 2

Fig. 2 Numerical solution and agglomerated meshes for different levels (Example 2).

HYPRE. A Library of High Performance Preconditioners. http://www . llnl.gov/CASC/hypre/.

George Karypis and Vipin Kumar. A fast and high quality multilevel scheme for partitioning irregular graphs. SIAM Journal on scientific Computing, 20(1):359-392, 1998.

T. V. Kolev and P. S. Vassilevski. Parallel auxiliary space AMG solver for H(div) problems. SIAM J. Sci. Comput., 34(6):A3079-A3098, 2012.

I. V. Lashuk and P. S. Vassilevski. Element agglomeration coarse RaviartThomas spaces with improved approximation properties. Numer. Linear Algebra Appl., 19(2):414-426, 2012.

I. V. Lashuk and P. S. Vassilevski. The construction of the coarse de Rham complexes with improved approximation properties. Comput. Methods Appl. Math., 14(2):257-303, 2014.

MFEM. Modular finite element methods. mfem.org.

J. E. Pasciak and P. S. Vassilevski. Exact de Rham sequences of spaces defined on macro-elements in two and three spatial dimensions. SIAM J. Sci. Comput., 30(5):2427-2446, 2008. 